\title{
A!
}

This is an electronic reprint of the original article.

This reprint may differ from the original in pagination and typographic detail.

Karttunen, Antti J.; Fässler, Thomas F.

\section{Semiconducting Clathrates Meet Gas Hydrates: Xe24[Sn136]}

Published in:

CHEMISTRY: A EUROPEAN JOURNAL

Published: 01/01/2014

Document Version

Peer reviewed version

Published under the following license:

Unspecified

Please cite the original version:

Karttunen, A. J., \& Fässler, T. F. (2014). Semiconducting Clathrates Meet Gas Hydrates: Xe24[Sn136].

CHEMISTRY: A EUROPEAN JOURNAL, 20(22), 6693-6698.

This material is protected by copyright and other intellectual property rights, and duplication or sale of all or part of any of the repository collections is not permitted, except that material may be duplicated by you for your research use or educational purposes in electronic or print form. You must obtain permission for any other use. Electronic or print copies may not be offered, whether for sale or otherwise to anyone who is not an authorised user. 


\title{
Semiconducting Clathrates Meet Gas Hydrates: $\mathbf{X e}_{24}\left[\mathbf{S n}_{136}\right]$
}

\author{
Antti J. Karttunen, ${ }^{*[a, b]}$ and Thomas F. Fässler*[c]
}

\begin{abstract}
Semiconducting group 14 clathrates are inorganic host-guest materials with a close structural relationship to gas hydrates. Here we utilize this inherent structural relationship to derive a new class of porous semiconductor materials: noble gas filled group 14 clathrates $\left(\mathrm{Ng}_{\mathrm{x}}\left[\mathrm{M}_{136}\right], \mathrm{Ng}=\mathrm{Ar}, \mathrm{Kr}, \mathrm{Xe}\right.$ and $\mathrm{M}=$ $\mathrm{Si}, \mathrm{Ge}, \mathrm{Sn})$. We have carried out highlevel quantum chemical studies using periodic Local-MP2 (LMP2) and
\end{abstract}

dispersion-corrected density functional methods (DFT-B3LYP-D) to properly describe the dispersive host-guest interactions. The adsorption of noble gas atoms within clathrate-II framework turned out to be energetically clearly favourable for several host-guest systems. For the energetically most favourable noble gas filled clathrate, $\mathrm{Xe}_{24}\left[\mathrm{Sn}_{136}\right]$, the adsorption energy is $-52 \mathrm{~kJ} / \mathrm{mol} /$ guest at the LMP2/TZVPP level of theory, corresponding to $-9.2 \mathrm{~kJ} / \mathrm{mol} / \mathrm{Sn}$ atom. Considering that a hypothetical guestfree Sn clathrate-II host framework is only $2.6 \mathrm{~kJ} / \mathrm{mol} / \mathrm{Sn}$ less stable than diamond-like $\alpha-\mathrm{Sn}$, the stabilization resulting from the noble gas adsorption is very significant.

Keywords: Semiconductors $\bullet$
Noble gases $\bullet$ Host-guest systems $\bullet$
Solid-state structures $\bullet$ Ab initio
calculations

\section{Introduction}

Semiconducting group 14 clathrates possess extraordinary electronic properties such as larger band gaps in comparison to the diamond structures of the respective elements ${ }^{[1,2]}$ and high thermoelectric efficiency. ${ }^{[3,4]}$ The semiconducting group 14 clathrates can be described as host-guest materials where tetrahedrally coordinated group 14 atoms form a framework composed of fused polyhedral cages. ${ }^{[5]}$ They are structurally closely related to gas hydrates (also known as gas clathrates or clathrate hydrates), where hydrogenbonded water molecules form a similar framework of polyhedral cages. ${ }^{[6]}$ Although the framework structures in the semiconducting clathrates and the gas hydrates are directly related to each other, they accommodate very different types of guest species. In the gas hydrates, typical guests within the polyhedral cages are small gaseous species such as methane or the noble gas atoms, which interact only weakly with the framework. In contrast, in the

[a] Dr. A. J. Karttunen

Department of Chemistry

Aalto University, FI-00076 Aalto, Finland

E-mail: antti.j.karttunen@iki.fi

[b] Dr. A. J. Karttunen

Department of Chemistry

University of Jyväskylä, FI-40014 Jyväskylä, Finland

[b] Prof. Dr. Thomas F. Fässler

Department of Chemistry

Technische Universität München

Lichtenbergstrasse 4, 85747 Garching, Germany

E-mail: Thomas.Faessler@1rz.tum.de semiconducting clathrates, the cages are typically occupied by alkaline, earth-alkaline, or halogen atoms and there is a strong ionic interaction between the guests and the tetrahedrally coordinated framework. ${ }^{[7]}$ However, in the case of cubic clathrate-II framework (Figure 1), practically "guest-free" modifications have been synthetized, ${ }^{[8-10]}$ in line with computational studies showing the clathrate-II framework to be the energetically the most favorable one within various experimentally observed and hypothetical clathrate frameworks. ${ }^{[11]}$ Recent synthesis of alloyed, guest-free SiGe clathrates is a promising step in the direction of tunable band gap materials for optoelectronic applications. ${ }^{[12,13]}$ In direct analogy to the gas hydrates, the existence of guest-free, uncharged semiconducting clathrates suggests that neutral gaseous species such as noble gas atoms or methane could be encapsulated within the semiconducting clathrates.

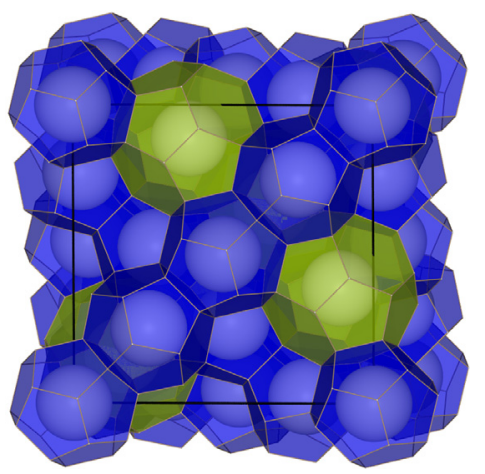

Figure 1. Cubic clathrate-II framework $(F d-3 m)$. The structure shown here is composed of fused 20-membered (blue) and 28-membered (green) atomic cages filled with guest atoms (136 tetrahedral framework atoms, 16 guests in the 20 -membered cages and 8 guests in the 28-membered cages). Unit cell edges are drawn in black. 
Semiconducting clathrates encapsulating weakly interacting guests are an intriguing alternative to the completely guest-free modifications of clathrates. For appropriate host-guest combinations, guest atoms such as the monatomic noble gas atoms could facilitate the formation of the clathrates without disrupting the electronic properties of the host framework. Interestingly, a related He-filled 20-membered carbon cage $\mathrm{He} @ \mathrm{C}_{20} \mathrm{H}_{20}$ has been prepared in small quantities, although the inclusion of a $\mathrm{He}$ atom inside the dodecahedrane is energetically very unfavorable (by about 140 $\mathrm{kJ} / \mathrm{mol}) .{ }^{[14,15]}$ Blasé et al. have previously investigated a Xe-filled clathrate-I structure $\mathrm{Xe}_{8}\left[\mathrm{Si}_{46}\right]$ using LDA-DFT calculations, but the inclusion of Xe inside the 20- and 24-membered cages in clathrate-I turned out to be energetically clearly unfavorable. ${ }^{[16,17]}$ However, quasiparticle band gap calculations on $\mathrm{Xe}_{8}\left[\mathrm{Si}_{46}\right]$ showed that the system still retained the wide band gap similar to the elemental clathrate modifications, suggesting that noble gas clathrates might in general possess very attractive electronic properties.

Here we investigate the adsorption of noble gas atoms inside semiconducting silicon, germanium, and tin clathrate-II frameworks using quantum chemical methods. The noble gas filled clathrates are a particularly challenging solid state system for theoretical studies due to the weak dispersion interactions between the guest atoms and the host framework. Since standard density functional (DFT) methods are unable to describe the weak dispersion interactions properly, we treat the electron correlation using state-of-the-art $a b$ initio local second-order Møller-Plesset perturbation theory (LMP2). ${ }^{[18]}$ Furthermore, the $a b$ initio LMP2 studies are accompanied by DFT studies using Grimme's empirical dispersion corrections (DFT-B3LYP-D3). ${ }^{[19,20]}$

\section{Results and Discussion}

Group 14 elements $\mathrm{Si}, \mathrm{Ge}$, and $\mathrm{Sn}$ are all known to form type II clathrates. ${ }^{[39-42]}$ For all three elements, the 20- and 28-membered cages are very different in size and obtaining clathrates with cages completely filled by 24 guest atoms requires careful consideration of the structural chemistry. Recent methodological advances have actually provided synthetic techniques to obtain stoichiometric typeII clathrates with one type of guest species such as $\mathrm{Na}_{24} \mathrm{Si}_{136},{ }^{[43]}$ but in the first completely filled $\mathrm{Si}\left(\mathrm{Cs}_{8} \mathrm{Na}_{16} \mathrm{Si}_{136}\right)$ and $\mathrm{Ge}$ $\left(\mathrm{Cs}_{8} \mathrm{Na}_{16} \mathrm{Ge}_{136}\right)$ clathrates, the key factor was to accommodate two very different-sized guest atoms in the differently sized 20- and 28membered cages. ${ }^{[39]}$

Considering the importance of the size factors in the synthesis of clathrate-II structures, we investigated two different noble gas adsorption schemes within the silicon, germanium, and tin type II clathrates. In the first scheme, only the larger 28 -membered cages of the clathrate framework are filled by the noble gas guests, resulting in 8 guests per unit cell (denoted as $\mathrm{Ng}_{8}\left[\mathrm{M}_{136}\right]$, where $\mathrm{Ng}=\mathrm{Ar}$, $\mathrm{Kr}$, $\mathrm{Xe}$ and $\mathrm{M}=\mathrm{Si}, \mathrm{Ge}, \mathrm{Sn}$ ). In the second scheme, both the 20- and 28membered cages are filled by the noble gas guest atoms, resulting in 24 guests per unit cell (denoted as $\mathrm{Ng}_{24}\left[\mathrm{M}_{136}\right]$ ). In the case of the silicon and germanium clathrates, already the $\mathrm{Kr}$ guests are much too large to fit in the smaller 20-membered cavities, and the completely filled $\mathrm{Xe}_{24}\left[\mathrm{Mi}_{136}\right]$ structures were not considered here. For tin, both the 20- and 28-membered cages are large enough to accompany all studied noble gas guest atoms and therefore we focused on the completely filled $\mathrm{Ng}_{24}\left[\mathrm{Sn}_{136}\right]$ variants. The noble gas atoms are located at the cage centers (crystallographic positions $16 \mathrm{c}$ and $8 b$ for the 20 - and 28 -membered cages, respectively). The adsorption energies per noble gas guest atom are reported in Table 1 at the LMP2/TZVPP and DFT-B3LYP-D3/TZVPP levels of theory.

Table 1. Adsorption energies $(\Delta E)$ in $\mathrm{kJ} / \mathrm{mol} /$ guest for the inclusion of noble gas guests inside the semiconducting clathrate-II host structures. ${ }^{[a]}$

\begin{tabular}{|c|c|c|}
\hline System $^{[b]}$ & $\Delta E_{\mathrm{LMP} 2}$ & $\Delta E_{\text {DFT-B3LYP-D3 }}$ \\
\hline $\mathrm{Ar}_{8}\left[\mathrm{Si}_{136}\right]$ & -33 & -41 \\
\hline $\mathrm{Ar}_{24}\left[\mathrm{Si}_{136}\right]$ & 4 & 20 \\
\hline $\mathrm{Kr}_{8}\left[\mathrm{Si}_{136}\right]$ & -53 & -55 \\
\hline $\mathrm{Kr}_{24}\left[\mathrm{Si}_{136}\right]$ & 13 & 46 \\
\hline $\mathrm{Xe}_{8}\left[\mathrm{Si}_{136}\right]$ & -79 & -77 \\
\hline $\mathrm{Ar}_{8}\left[\mathrm{Ge}_{136}\right]$ & -32 & -41 \\
\hline $\mathrm{Ar}_{24}\left[\mathrm{Ge}_{136}\right]$ & -6 & 2 \\
\hline $\mathrm{Kr}_{8}\left[\mathrm{Ge}_{136}\right]$ & -50 & -55 \\
\hline $\mathrm{Kr}_{24}\left[\mathrm{Ge}_{136}\right]$ & -5 & 19 \\
\hline $\mathrm{Xe}_{8}\left[\mathrm{Ge}_{136}\right]$ & -78 & -82 \\
\hline $\mathrm{Ar}_{8}\left[\mathrm{Sn}_{136}\right]$ & -21 & -32 \\
\hline $\mathrm{Ar}_{24}\left[\mathrm{Sn}_{136}\right]$ & -19 & -29 \\
\hline $\mathrm{Kr}_{8}\left[\mathrm{Sn}_{136}\right]$ & -35 & -48 \\
\hline $\mathrm{Kr}_{24}\left[\mathrm{Sn}_{136}\right]$ & -33 & -35 \\
\hline $\mathrm{Xe}_{8}\left[\mathrm{Sn}_{136}\right]$ & -62 & -73 \\
\hline $\mathrm{Xe}_{24}\left[\mathrm{Sn}_{136}\right]$ & -52 & -54 \\
\hline
\end{tabular}

[a] The adsorption is energetically favorable for $\Delta E<0$ and unfavorable for $\Delta E>0$. The LMP2/TZVPP and DFT-B3LYP-D3/TZVPP adsorption energies have been Counterpoise-corrected for basis set superposition error. [b] In the case of the $\mathrm{Ng}_{8}\left[\mathrm{M}_{136}\right]$ systems, only the larger 28-membered cages are occupied by guests. $\mathrm{Xe}_{24}\left[\mathrm{Si}_{136}\right]$ and $\mathrm{Xe}_{24}\left[\mathrm{Ge}_{136}\right]$ were not included in the study because the adsorption of Xe atoms inside the $\mathrm{Si}_{20}$ and $\mathrm{Ge}_{20}$ cages is energetically very unfavourable.

The adsorption of noble gas atoms within the clathrate-II framework turned out to be energetically clearly favourable for several host-guest systems. To make the adsorption energies in Table 1 more concrete, it is helpful to compare how the overall stability of the clathrate framework changes with respect to diamond-like bulk when the noble gas atoms adsorb within the framework. In the following comparisons, the relative energies of the empty clathrate-II frameworks with respect to the bulk $\alpha$-phases have been obtained at the DFT-PBE0/SVP level of theory, and they are in good agreement with previous theoretical studies. ${ }^{[11]}$ In the case of $\mathrm{Xe}_{24}\left[\mathrm{Sn}_{136}\right]$, the LMP2 adsorption energy of -52 $\mathrm{kJ} / \mathrm{mol} /$ guest is equivalent to $-1248 \mathrm{~kJ} / \mathrm{mol} / \mathrm{unit}$ cell or -9.2 $\mathrm{kJ} / \mathrm{mol} / \mathrm{Sn}$ atom. Considering that the hypothetical guest-free $\mathrm{Sn}$ clathrate-II framework is only $2.6 \mathrm{~kJ} / \mathrm{mol} / \mathrm{Sn}$ less stable than the diamond-like $\alpha$-Sn, the additional stabilization resulting from the noble gas adsorption is very significant. In other words, the noble gas adsorption could facilitate the experimental realization of a porous, semiconducting $\mathrm{Sn}$ framework (entropic contributions are discussed below). For the $\mathrm{Ar}_{24}\left[\mathrm{Sn}_{136}\right]$ and $\mathrm{Kr}_{24}\left[\mathrm{Sn}_{136}\right]$ systems, the LMP2 adsorption energies of -19 and $-33 \mathrm{~kJ} / \mathrm{mol} /$ guest are equivalent to -3.4 and $-5.8 \mathrm{~kJ} / \mathrm{mol} / \mathrm{Sn}$, respectively, making these systems also energetically favourable in comparison to $\alpha$-Sn. For $\mathrm{Xe}_{8}\left[\mathrm{Si}_{136}\right]$ and $\mathrm{Xe}_{8}\left[\mathrm{Ge}_{136}\right]$, the adsorption energies of -78 and -79 $\mathrm{kJ} / \mathrm{mol} /$ guest are equivalent to $-4.6 \mathrm{~kJ} / \mathrm{mol} /$ framework atom. For comparison, the empty $\mathrm{Si}$ and Ge clathrate-II frameworks are 8.1 and $3.7 \mathrm{~kJ} / \mathrm{mol} /$ atom less stable than $\alpha$-Si and $\alpha$-Ge, respectively. Thus, partial noble gas adsorption would not be enough to stabilize the Si clathrate-II framework with respect to $\alpha-\mathrm{Si}$, but in the case of germanium Xe adsorption in the larger 28-membered cages could 
stabilize the clathrate-II framework. Finally, while the clathrate-II framework is the energetically most stable among all empty group 14 clathrate frameworks, the noble gas adsorption could be energetically even more favourable for some other clathrate frameworks with different constituent cages. ${ }^{[1]}$

Concerning the applied theoretical methods, the adsorption energy trends obtained at the LMP2 and DFT-B3LYP-D3 levels of theory are generally in good agreement, the only cases of qualitative disagreement being $\mathrm{Ar}_{24}\left[\mathrm{Ge}_{136}\right]$ and $\mathrm{Kr}_{24}\left[\mathrm{Ge}_{136}\right]$, where LMP2 predicts adsorption to be favourable by a small margin, while DFTB3LYP-D3 predicts the adsorption to be unfavourable. Also for $\mathrm{Kr}_{24}\left[\mathrm{Si}_{136}\right]$, where the adsorption is clearly unfavourable with both methods, the quantitative difference of the adsorption energies is rather large. For the $\mathrm{Ng}_{8}\left[\mathrm{Si}_{136}\right]$ systems, where only the larger 28membered cage is occupied, the LMP2 and DFT-B3LYP-D3 methods agree well. For the $\mathrm{Ge}_{136}$ and $\mathrm{Sn}_{136}$ clathrates, the LMP2 and DFT-B3LYP-D3 results show very similar trends, DFTB3LYP-D3 predicting somewhat larger adsorption energies for all cases where the adsorption is energetically favourable. The overall trends from the LMP2 and DFT-B3LYP-D3 adsorption energies are very clear: for $\mathrm{Si}_{136}$ and $\mathrm{Ge}_{136}$, the full occupation of all 24 cages is only favourable at the LMP2 level of theory for $\mathrm{Ar}_{24}\left[\mathrm{Ge}_{136}\right]$ and $\mathrm{Kr}_{24}\left[\mathrm{Ge}_{136}\right]$ and even in these cases with a rather small margin. The occupation of the larger 28-membered cages is always favoured for all investigated combinations of clathrate-II frameworks and noble gases, Xe showing the highest adsorption energies. Furthermore, for $\mathrm{Sn}_{136}$, the complete occupation of the 20- and 28-membered cages is energetically favourable by a clear margin for all studied noble gas atoms. The LMP2 adsorption energy for the Xe atoms in the 20membered cages of $\mathrm{Sn}_{136}$ is $-49 \mathrm{~kJ} / \mathrm{mol} /$ guest.

The MP2 method is known to overestimate weak van der Waals interaction in many cases. Furthermore, even though the periodic LMP2 calculations included here are already at the limit of feasibility with the present serial version of CRYSCOR09, the applied TZVPP basis set is still far from the basis set limit. A previous systematic investigation of $\mathrm{Ng}$ atoms encapsulated inside a $\mathrm{C}_{60}$ fullerene showed that canonical MP2 calculations with a triplevalence-zeta level basis set overestimated the host-guest interaction in comparison to high-level DFT-SAPT results (symmetry-adapted perturbation). ${ }^{[44][45]}$ The same study also showed the older dispersion-corrected DFT-D2 implementation to overestimate the dispersion interactions for the $\mathrm{Ng} @ \mathrm{C}_{60}$ systems. Concerning the basis set completeness for periodic LMP2 calculations, Maschio et al. have shown that for molecular crystal such as solid $\mathrm{NH}_{3}$ and $\mathrm{CO}_{2}$, relatively diffuse high angular momentum basis functions are necessary to reach the basis set limit. ${ }^{[46]}$ While such diffuse basis functions often lead into numerical difficulties in periodic HF and DFT calculations, they can be included in periodic LMP2 calculations by means of dual basis set technique, where the HF reference is calculated using a smaller basis set. Here we were limited by the computational feasibility of the LMP2 calculations and did not take advantage of the dual basis set technique.

To test the basis set completeness of the periodic calculations, we carried out molecular benchmark calculations, where much larger basis sets could be utilized. The utilization of molecular reference systems is a very convenient approach in the cases where suitable finite model systems are available. For example, highquality physisorption energies for argon monolayers adsorbed on the $\mathrm{MgO}$ (100) surface have been obtained by scaling periodic LMP2 energetics based on molecular $\operatorname{CCSD}(\mathrm{T})$ calculations extrapolated to the basis set limit. ${ }^{[4]}$ We derived a suitable molecular benchmark system by extracting one 28 -membered cage from the clathrate-II structure and saturating it with hydrogen atoms. The noble gas adsorption energies within the resulting $\mathrm{M}_{28} \mathrm{H}_{28}$ cage can then be compared with the results obtained for the bulk clathrate-II structure. Since the adsorption of the noble gas atoms inside the 28-membered cages is clearly energetically favourable for all $\mathrm{Ng}-\mathrm{M}$ combinations, the adsorption energies can be compared on the same footing. Naturally, replacing one $\mathrm{M}-\mathrm{M}$ bond with an $\mathrm{M}-\mathrm{H}$ bond slightly affects the polarizability of the $\mathrm{M}$ atoms, which in turn can have a minor effect on the dispersive interactions between the noble gas atom and the cage. Therefore, the adsorption energies for the periodic $\mathrm{M}_{28}$ cage and the molecular $\mathrm{M}_{28} \mathrm{H}_{28}$ cage are not expected to be identical even at the completely same level of theory. The molecular adsorption energies at the MP2/QZVPP and DFTB3LYP-D3/QZVPP levels of theory are listed in table S2 in the Supporting Information. Higher-level molecular benchmarks using $\operatorname{CCSD}(\mathrm{T})$ method turned out to be computationally impractical at the moment, but in further studies on the noble-gas filled clathrates the DFT-SAPT approach mentioned above could be used as a higher-level reference. ${ }^{[44][45]}$

The comparisons between the adsorption energies of the periodic and molecular cases show that for the DFT-B3LYP-D3 method the TZVPP basis set is already rather close to the basis set limit, while in the case of the MP2 method the adsorption energies increase clearly when the basis set is enlarged from TZVPP to def2QZVPP. Overall, the MP2/QZVPP and DFT-B3LYP-D3/QZVPP adsorption energies from the molecular benchmark calculations are in very similar and only in two cases, $\mathrm{Xe}_{8}\left[\mathrm{Si}_{28}\right]$ and $\mathrm{Xe}_{8}\left[\mathrm{Ge}_{28}\right]$, the difference between them is larger than $10 \%$ (but still smaller than $20 \%$ ). The dispersion corrections obtained with DFT-D3 have been previously shown to be of very high quality across the periodic table ${ }^{[19,20]}$ and the good agreement of the molecular MP2/QZVPP and B3LYP-D3/QZVPP adsorption energies is encouraging considering the overall accuracy of the LMP2 adsorption energies listed in Table 1. The periodic LMP2/TZVPP adsorption energies are consistently smaller than the molecular MP2/QZVPP adsorption energies, suggesting that the LMP2/TZVPP adsorption energies of Table 1 would become more favourable if was computationally feasible to carry out the periodic LMP2 calculations with even larger basis sets. The DFT-B3LYP-D3/TZVPP level of theory is a very promising combination for further studies on the noble-gas filled clathrates since it also enables accurate dispersion-corrected geometry optimizations and frequency calculations in the solid state.

The LMP2 or DFT-B3LYP-D3 methods make it possible to study the energetics of the dispersive $\mathrm{Ng}-\mathrm{M}$ interactions, but they cannot be used to evaluate how the noble gas encapsulation affects the electronic structure of the group 14 clathrates. To gain a qualitative understanding of the electronic structure of the $\mathrm{Ng}_{\mathrm{x}}\left[\mathrm{M}_{136}\right]$ systems, we analysed their Density of States (DOS) at the HF/TZVPP level of theory. It turned out that that the valence energy levels of the noble gas atoms are well below the valence bands of the group 14 frameworks (Figure S2 in Supporting information). The noble gas adsorption is therefore expected to have practically no effect on the electronic properties of the group 14 clathrate frameworks, which are wide band gap semiconductors. ${ }^{[11]}$

For a more detailed understanding of the size effects of the noble gas guests, Table 2 lists van der Waals radii based "filling 
ratios" for all combinations of polyhedral cages and noble gas atoms. Comparison of the filling ratios and the adsorption energies suggests that the energy balance between the attractive dispersion interaction and repulsive interactions due to the guest is very delicate: when the filling ratio reaches $\sim 1.2$, the noble gas adsorption inside the cage becomes unfavourable.

Table 2. Structural details of the studied $\mathrm{Ng}_{x}\left[\mathrm{M}_{136}\right]$ systems in terms of the constituent cages.

\begin{tabular}{lllll} 
Cage & $R_{\mathrm{Ng}-\mathrm{M}}(\AA)^{[\mathrm{a}]}$ & Ar Filling ratio $^{[\mathrm{b}]}$ & Kr Filling ratio & Xe Filling ratio \\
\hline $\mathrm{Si}_{20}$ & 3.19 & 1.25 & 1.29 & 1.34 \\
$\mathrm{Ge}_{20}$ & 3.33 & 1.20 & 1.24 & 1.28 \\
$\mathrm{Sn}_{20}$ & 3.81 & 1.06 & 1.10 & 1.14 \\
$\mathrm{Si}_{28}$ & 3.93 & 1.01 & 1.05 & 1.08 \\
$\mathrm{Ge}_{28}$ & 4.11 & 0.97 & 1.00 & 1.04 \\
$\mathrm{Sn}_{28}$ & 4.71 & 0.86 & 0.89 & 0.92 \\
\hline
\end{tabular}

[a] The shortest $\mathrm{Ng}-\mathrm{M}$ distance within the cage. [b] The filling ratio is defined as the ratio of the sum of the van der Waals radii of $\mathrm{Ng}$ and $\mathrm{M}$ and the shortest $\mathrm{Ng}-\mathrm{M}$ distance $\left(R_{\mathrm{Ng}-\mathrm{M}}\right)\left(F R=\left(\mathrm{R}_{\mathrm{vdW}}(\mathrm{Ng})+\mathrm{R}_{\mathrm{vdW}}(\mathrm{M})\right) / R_{\mathrm{Ng}-\mathrm{M}}\right.$. If $\mathrm{FR}>1$, the sum of the van der Waals radii exceeds the shortest $\mathrm{Ng}-\mathrm{M}$ distance. Applied van der Waals radii $(\AA): \mathrm{r}(\mathrm{Si})=2.10$; $r(\mathrm{Ge})=2.11 ; r(\mathrm{Sn})=2.17 ; r(\mathrm{Ar})=1.88 ; r(\mathrm{Kr})=2.02 ; r(\mathrm{Xe})=2.16{ }^{[48]}$

Overall, the energy comparisons between the noble gas filled clathrates and the $\alpha$-phases look very promising, but for their experimental realization, thermodynamics and in particular entropic effects need to be considered, too. Although we could not carry out a complete thermodynamic analysis due to the large size of the systems and the unavailability of LMP2 frequency calculations, it is clear that confining the noble gas atoms inside the polyhedral cages will result in loss of translational entropy. The standard molar entropy of $\mathrm{Xe}$ is $169.68 \mathrm{~J} /(\mathrm{K} \mathrm{mol})$ and assuming that the encapsulated Xe atoms would lose all their translational entropy, the total TS contribution to the Gibbs free energy would be 51 $\mathrm{kJ} / \mathrm{mol} /$ guest at $298 \mathrm{~K}$. At this temperature, the predicted LMP2 $\Delta E$ value of $-52 \mathrm{~kJ} / \mathrm{mol} /$ guest for the adsorption of Xe within the clathrate-II structure is still exoergic enough that the total Gibbs free energy of the encapsulation should remain exothermic, but as the conventional strategies for clathrate synthesis occur in rather high temperatures, the entropy loss could become more significant and exceed the energy gains from the adsorption.

In the preceding thermodynamic analysis we assumed that the $\mathrm{Xe}$ atoms lose all their translational entropy when encapsulated in $\mathrm{Xe}_{24}\left[\mathrm{Sn}_{136}\right]$, but in reality the $\mathrm{Xe}$ atoms would have some room to vibrate inside the Sn cages, increasing the entropy of the gas-filled clathrate. To gain further insights on the thermodynamics of $\mathrm{Xe}_{24}\left[\mathrm{Sn}_{136}\right]$ with respect to $\alpha$-Sn, we carried out few molecular reference calculations (DFT-D3 dispersion corrections can be combined with analytical second derivatives in the TURBOMOLE program package). We used hydrogen-terminated $\mathrm{Sn}_{20} \mathrm{H}_{20}$ and $\mathrm{Sn}_{28} \mathrm{H}_{28}$ cages as models for the 20- and 28-membered cages in the $\mathrm{Sn}_{136}$ clathrate and calculated the vibrational frequencies of the $\mathrm{Xe}\left[\mathrm{Sn}_{20} \mathrm{H}_{20}\right]$ and $\mathrm{Xe}\left[\mathrm{Sn}_{28} \mathrm{H}_{28}\right]$ systems at the B3LYP-DFT-D3/def2TZVPP level of theory. The $\Delta E$ values for encapsulating $\mathrm{Xe}$ atom in $\mathrm{Sn}_{20} \mathrm{H}_{20}$ and $\mathrm{Sn}_{28} \mathrm{H}_{28}$ are -49 and $-83 \mathrm{~kJ} / \mathrm{mol} /$ guest, while the corresponding $\Delta G$ values are -1 and $-47 \mathrm{~kJ} / \mathrm{mol} /$ guest $(T=298 \mathrm{~K}, p$ $=1 \mathrm{~atm})$. Using the molecular $\Delta G$ values, the total $\Delta G$ for encapsulating $24 \mathrm{Xe}$ atoms within $\mathrm{Sn}_{136}$ would be $-384 \mathrm{~kJ} / \mathrm{mol}$, which is equal to $-2.8 \mathrm{~kJ} / \mathrm{mol} / \mathrm{Sn}$ atom. This is again very close to the $2.6 \mathrm{~kJ} / \mathrm{mol} / \mathrm{Sn}$ energy difference between the empty Sn clathrate-
II framework and diamond-like $\alpha-\operatorname{Sn}(\Delta E$ and $\Delta G$ are expected to be rather similar when comparing such solid state species). The above molecular reference calculations do not exactly reflect the situation for real $\mathrm{Xe}_{24}\left[\mathrm{Sn}_{136}\right]$, but the thermodynamic estimates are very encouraging from the point of view of experimental synthesis and using high noble gas pressures could further stabilize $\mathrm{Xe}_{24}\left[\mathrm{Sn}_{136}\right]$ with respect to $\mathrm{Xe}(\mathrm{g})$ and $\alpha-\mathrm{Sn}$.

An interesting experimental strategy towards the noble gas filled clathrates would be the utilization of ionic liquids. Ionic liquids have already been used to synthesize the practically guest-free Ge clathrate-II via oxidation of $\mathrm{Ge}_{9}{ }^{4-}$ Zintl anions. ${ }^{[10]}$ Noble gas atoms are readily soluble in ionic liquids ${ }^{[49,50]}$ and if the oxidation of the $\mathrm{M}_{9}{ }^{4-}$ anions is carried out in an ionic liquid with a noble gas high concentration, the translational entropy of the noble gas atoms has already been greatly reduced during the solvation to the ionic liquid. Hence, the entropy loss due inclusion in the clathrate structure would not be so drastic and the noble gas inclusion within the clathrate framework could become thermodynamically favoured.

The inclusion of gas molecules within semiconducting clathrates has been shown to be possible with the existing experimental methods: hydrogen molecules were reported to encapsulate within the clathrate-I structure $\mathrm{Na}_{5.5}\left(\mathrm{H}_{2}\right)_{2.15} \mathrm{Si}_{46}$, which is stable at ambient temperature and pressure. ${ }^{[51]}$ The synthesis was carried out by reacting $\mathrm{NaSi}$ and $\mathrm{NH}_{4} \mathrm{Br}$ under dynamic vacuum at $300{ }^{\circ} \mathrm{C}$. Similarly to $\mathrm{Na}_{5.5}\left(\mathrm{H}_{2}\right)_{2.15} \mathrm{Si}_{46}$, the noble gas atoms could also coexist in a clathrate structure with other types of guest atoms such as alkali metals. Generally, vacuum treatment of the clathrate structures has been shown to allow efficient manipulation of the host-guest structure, as the practically guest-free Si clathrate-II structure could be prepared via ultra-high vacuum treatment of $\mathrm{Na}_{\mathrm{x}} \mathrm{Si}_{136} \cdot{ }^{\left[{ }^{[]}\right.}$

\section{Conclusion}

We have investigated semiconducting group 14 clathrate-II structures filled with noble gas atoms. Quantum chemical calculations at the LMP2/TZVPP and DFT-B3LYP-D3/TZVPP levels of theory showed the inclusion of noble gas atoms within the clathrate-II framework to be energetically favourable. The adsorption energy for the energetically most favourable noble gas filled clathrate, $\mathrm{Xe}_{24}\left[\mathrm{Sn}_{136}\right]$, is $-52 \mathrm{~kJ} / \mathrm{mol} / \mathrm{guest}$ at the LMP2/TZVPP level of theory. This corresponds to $-9.2 \mathrm{~kJ} / \mathrm{mol} / \mathrm{Sn}$ atom, which is an exciting finding considering that previous DFT studies have shown the hypothetical guest-free Sn clathrate-II framework to be only $2.6 \mathrm{~kJ} / \mathrm{mol} / \mathrm{Sn}$ less stable than $\alpha$-Sn. Due to the non-intrusive nature of the noble gas guests, the experimental realization of the noble gas filled group 14 clathrates would result in a new class of wide band gap semiconductor materials.

\section{Experimental Section}

The noble gas filled clathrate-II structures were investigated using the local secondorder Møller-Plesset perturbation theory (LMP2) method as implemented in the CRYSCOR09 software. ${ }^{[18]}$ The Hartree-Fock wavefunction and the (symmetrized) localized Wannier functions (WFs) necessary for the LMP2 procedure were obtained with the CRYSTAL09 program package. ${ }^{[21,22]}$ CRYSTAL09 was also used to carry out density functional calculations at the DFT-B3LYP-D $3^{[23-25]}$ level of theory using Grimme's D3 empirical dispersion correction. ${ }^{[19,20]}$ The CRYSTAL09 and CRYSCOR09 programs utilize localized atomic basis sets composed of Gaussian-type functions. We applied triple-zeta-valence + extended polarization (TZVPP) level basis sets in both LMP2 and DFT-B3LYP-D3 calculations. 10-, 28-, and 28-electron scalarrelativistic pseudopotentials were used to describe the core electrons of $\mathrm{Ge}, \mathrm{Sn}$, and $\mathrm{Xe}$, respectively. The basis sets were derived from the molecular Karlsruhe basis sets, ${ }^{[26,27]}$ 
detailed basis set listings are given in the Supporting information. The clathrate framework geometries, optimized at the PBE0/SVP level of theory, were taken from our previous study on group 14 clathrate frameworks. ${ }^{[11]}$ The lattice constant $a$ is 14.72 , 15.36, and $17.61 \AA$ for $\mathrm{Si}, \mathrm{Ge}$, and Sn clathrate-II frameworks, respectively. As the current public version of CRYSCOR does not provide analytical gradients for the LMP2 method, we did not consider any structural relaxation for the noble-gas filled clathrate frameworks. However, in the case of dispersion-corrected DFT-B3LYP calculations, we also investigated the effect of structural relaxation due to the noble gas guests (details given below).

In addition to the periodic solid-state calculations, we carried out molecular benchmark calculations at MP2/def2-QZVPP and DFT-B3LYP-D3/def2-QZVPP levels of theory using the TURBOMOLE program package (version 6.5). ${ }^{[27-30]}$

For accurate LMP2 calculations, a number of technical keywords have to be carefully considered for the Hartree-Fock step, WF generation, and the actual LMP2 step. In al steps, a shrinking factor of 4 (SHRINK) was used to generate a Monkhorst-Pack-type grid of $k$-points in the reciprocal space, resulting in $8 k$-points in the irreducible Brillouin zone of the clathrate-II structure. ${ }^{[31]}$ For the evaluation of the Coulomb and exchange integrals during the HF step (TOLINTEG), very tight tolerance factors were applied (Si-clathrates: 8, 8, 8, 16, 50; Ge/Sn-clathrates: 8, 8, 8, 16, 40). Tight SCF convergence criterion was also applied in the HF calculations (TOLDEE 9). In the localization of the Wannier functions, only the WFs arising from the valence bands were considered. When symmetrizing the WFs for the LMP2 calculation, the tolerance for finding a homoatomic bond (OMOBOND) was increased to $0.1 .^{[32,33]}$ In the LMP2 calculations, we utilized the direct-space density-fitting techniques to avoid the calculation of the exact two-electron four-center integrals. ${ }^{[34]}$ The density-fitting technique, also known as the "resolution-of-identity approximation", requires an auxiliary basis set in addition to the standard orbital basis set. We applied TZVPP-level fitting basis sets derived from the molecular Karlsruhe RI-MP2 auxiliary basis sets, ${ }^{[29,30]}$ detailed basis set listings are given in the Supporting information.

In the periodic LMP2 approach, the local $\left(R^{-6}\right)$ nature of electron correlation is exploited in two ways: The virtual space is truncated by means of so-called WF-pair domains and the number of interacting WF pairs is truncated by means of direct-space cutoffs. ${ }^{[18}$ Here, the excitation domains were defined with the DOMDEF2 approach: the eight nearest group 14 atoms were included in the excitation domain of each bonding WF between two group 14 atoms, while for the atomic WFs of the noble gas atoms it was enough to only include the noble gas atom itself in the excitation domain. Since the present work deals with the adsorption of gas atoms inside a 3D framework, the WF pair distance cutoffs were defined utilizing the very convenient pair partitioning scheme, where the system is divided into "environment" (the clathrate framework) and "molecule" (the noble gas atoms). In the calculation of the adsorption energies, no molecule-molecule or environment-enviroment WF pairs are allowed to interact, so that the LMP2 correlation energy arises from molecule-environment interactions only. However, we also checked the magnitude of the interactions between the noble gas atoms. In the case of $\mathrm{Ar}_{8}\left[\mathrm{Si}_{136}\right]$ ( $\mathrm{Ar}-\mathrm{Ar}$ distance $6.38 \AA$ ), the LMP2 correlation energy between two Ar atoms turned out to be only $1 \mathrm{~kJ} / \mathrm{mol}$. The density-fitting and multipole moment cutoffs $d_{1}$ and $d_{2}$ were set to 6 and $10 \AA$, respectively (after confirming the convergence with respect to longer cutoff distances of $d_{1}=8$ and $d_{2}=12 \AA$ ).

The local correlation methods are known to show much smaller basis set superposition error (BSSE) in comparison to their canonical counterparts, ${ }^{[35]}$ and in fact the moleculeenvironment pair partitioning scheme described above results in practically BSSE-free LMP2 adsorption energies. However, the underlying HF/TZVPP energies still show a minor BSSE contribution and we have corrected them using the Counterpoise scheme. ${ }^{[36]}$ LMP2 and DFT-B3LYP-D3 adsorption energies reported in Table 1 include Counterpoise corrections. Non-corrected adsorption energies have been listed in the Supporting Information (Table S1).

For the DFT-B3LYP-D3 calculations, we applied the Becke-Johnson damping scheme and the parameter sets released in August 2013 (V3.0 Rev 2). ${ }^{[19,20]}$ The DFT-D3 dispersion corrections were calculated with the stand-alone $d f t d 3$ program package available from the Grimme group. The CRYSTAL09 program package includes an implementation of the older DFT-D2 methodology (with analytic gradients), which we used to check the effect of the structural relaxation due to the noble gas guests for the $\mathrm{Ar}_{24}\left[\mathrm{Si}_{136}\right]$ structure. ${ }^{[37]}$ During the geometry optimization, the lattice constant increased by $1.3 \%$ and the adsorption energy changed from 30 to $29 \mathrm{~kJ} / \mathrm{mol} /$ guest. Since the effect of the structural relaxation was very small, we report the adsorption energies withou any effects of structural relaxation. For the DFT-D2 calculation, the scaling factor $\left(s_{6}\right)$ steepness of the damping function $(d)$, and the cutoff for direct lattice summation $\left(R_{c u t}\right)$ were set to $1.05,20$, and $25 \AA$, respectively. The atomic $C_{6}$ coefficients and van der Waals radii were taken directly from Grimme's 2006 dataset. ${ }^{[38]}$ In all DFT-B3LYP calculations, TOLINTEG was set to a tight value of $8,8,8,8,16$ and an extra-large integration grid (XLGRID) was applied.

\section{Acknowledgements}

A. J. K thanks Dr. Silvia Casassa and Dr. Lorenzo Maschio (University of Turin) for helpful discussions and for providing modified versions of the CRYSTAL09 and CRYSCOR09 binaries, respectively. We gratefully acknowledge funding from the Academy of Finland (A. J. K., grant 138560/2010) and Deutsche Forschungsgemeinschaft (T. F. F., DFG project numbers FA 198/11-1). The Finnish IT Center for Science (CSC) is thanked for providing the computing resources for this work.

[1] G. B. Adams, M. O'Keeffe, A. A. Demkov, O. F. Sankey, Y. Huang, Phys. Rev. B 1994, 49, 8048-8053.

[2] J. Dong, O. F. Sankey, J. Phys. : Condens. Matter 1999, 11, 6129-6145.

[3] G. S. Nolas, J. L. Cohn, G. A. Slack, S. B. Schujman, Appl. Phys. Lett. 1998, 73, 178-180.

[4] M. Christensen, S. Johnsen, B. B. Iversen, Dalton Trans. 2010, 39, 978-992.

[5] A. Shevelkov, K. Kovnir, Struct. Bond. 2011, 139, 97-142.

[6] G. A. Jeffrey, J. Inclusion Phenom. 1984, 1, 211-222.

[7] C. Gatti, L. Bertini, N. P. Blake, B. B. Iversen, Chem. Eur. J. 2003, 9, 4556-4568.

[8] J. Gryko, P. F. McMillan, R. F. Marzke, G. K. Ramachandran, D. Patton, S. K. Deb, O. F. Sankey, Phys. Rev. B 2000, 62, R7707.

[9] A. Ammar, C. Cros, M. Pouchard, N. Jaussaud, J. Bassat, G. Villeneuve, M. Duttine, M. Ménétrier, E. Reny, Solid State Sci. 2004, 6, 393-400.

[10] A. M. Guloy, R. Ramlau, Z. Tang, W. Schnelle, M. Baitinger, Y. Grin, Nature 2006, 443, 320-323

[11] A. J. Karttunen, T. F. Faessler, M. Linnolahti, T. A. Pakkanen, Inorg. Chem. 2011 $50,1733-1742$

[12] A. D. Martinez, L. Krishna, L. L. Baranowski, M. T. Lusk, E. S. Toberer, A. C. Tamboli, IEEE Journal of Photovoltaics, 2013, 3, 1305-1310.

[13] L. L. Baranowski, L. Krishna, A. D. Martinez, T. Raharjo, V. Stevanovic, A. C. Tamboli, E. S. Toberer, J. Mater. Chem. C 2014, In press, DOI: $10.1039 /$ C3TC32102A.

[14] R. Cross, M. Saunders, H. Prinzbach, Org. Lett. 1999, 1, 1479-1481.

[15] H. Jimenez-Vazquez, J. Tamariz, R. Cross, J. Phys. Chem. A 2001, 105, 1315-1319.

[16] D. Connétable, V. Timoshevskii, E. Artacho, X. Blase, Phys. Rev. Lett. 2001, 87, 206405.

[17] X. Blase, Phys. Rev. B 2003, 67, 035211.

[18] C. Pisani, M. Schuetz, S. Casassa, D. Usvyat, L. Maschio, M. Lorenz, A. Erba, Phys. Chem. Chem. Phys. 2012, 14, 7615-7628.

[19] S. Grimme, J. Antony, S. Ehrlich, H. Krieg, J. Chem. Phys. 2010, 132, 154104

[20] S. Grimme, S. Ehrlich, L. Goerigk, J. Comput. Chem. 2011, 32, 1456-1465.

[21] R. Dovesi, R. Orlando, B. Civalleri, C. Roetti, V. R. Saunders, C. M. ZicovichWilson, Z. Kristallogr. 2005, 220, 571-573.

[22] R. Dovesi, V. R. Saunders, R. Roetti, R. Orlando, C. M. Zicovich-Wilson, F. Pascale, B. Civalleri, K. Doll, N. M. Harrison, I. J. Bush, P. D’Arco, M. Llunell, CRYSTALO9 User's Manual. University of Torino: Torino, Italy 2009,.

[23] A. D. Becke, J. Chem. Phys. 1993, 98, 5648-5652.

[24] C. T. Lee, W. T. Yang, R. G. Parr, Phys. Rev. B 1988, 37, 785-789.

[25] P. J. Stephens, F. J. Devlin, C. F. Chabalowski, M. J. Frisch, J. Phys. Chem. 1994 98, 11623-11627.

[26] A. Schaefer, C. Huber, R. Ahlrichs, J. Chem. Phys. 1994, 100, 5829-5835.

[27] F. Weigend, R. Ahlrichs, Phys. Chem. Chem. Phys. 2005, 7, 3297-3305.

[28] R. Ahlrichs, M. Baer, M. Haeser, H. Horn, C. Koelmel, Chem. Phys. Lett. 1989, 162, 165-169.

[29] F. Weigend, M. Haeser, H. Patzelt, R. Ahlrichs, Chem. Phys. Lett. 1998, 294, $143-$ 152 .

[30] A. Hellweg, C. Haettig, S. Hoefener, W. Klopper, Theor. Chem. Acc. 2007, 117, 587-597.

[31] H. J. Monkhorst, J. D. Pack, Phys. Rev. B 1976, 13, 5188.

[32] C. Zicovich-Wilson, R. Dovesi, V. Saunders, J. Chem. Phys. 2001, 115, 9708-9719. 
[33] S. Casassa, C. M. Zicovich-Wilson, C. Pisani, Theor Chem. Acc 2006, 116, 726733.

[34] L. Maschio, D. Usvyat, Phys. Rev. B 2008, 78, 073102.

[35] M. Schuetz, G. Rauhut, H. Werner, J Phys Chem A 1998, 102, 5997-6003.

[36] S. F. Boys, F. Bernardi, Mol. Phys. 1970, 19, 553-566.

[37] B. Civalleri, C. M. Zicovich-Wilson, L. Valenzano, P. Ugliengo, CrystEngComm 2008, 10, 405-410.

[38] S. Grimme, J. Comput. Chem. 2006, 27, 1787-1799.

[39] S. Bobev, S. Sevov, J. Am. Chem. Soc. 1999, 121, 3795-3796.

[40] S. Bobev, S. Sevov, J. Solid State Chem. 2000, 153, 92-105.

[41] M. C. Schaefer, S. Bobev, J. Am. Chem. Soc. 2013, 135, 1696-1699.

[42] R. Kroner, K. Peters, H. G. von Schnering, R. Nesper, Z. Krist. New Cryst. St. 1998, 213, 664-664.

[43] S. Stefanoski, M. Beekman, W. Wong-Ng, P. Zavalij, G. S. Nolas, Chem. Mater. 2011, 23, 1491-1495.

[44] A. Hesselmann, T. Korona, Phys. Chem. Chem. Phys. 2011, 13, 732-743.
[45] A. Heßelmann, G. Jansen, M. Schütz, J. Chem. Phys. 2005 122, 014103.

[46] L. Maschio, D. Usvyat, M. Schütz, B. Civalleri, J. Chem. Phys. 2010 132, 134706.

[47] D. Usvyat, K. Sadeghian, L. Maschio, M. Schuetz, Phys. Rev. B 2012, 86, 045412.

[48] M. Mantina, A. C. Chamberlin, R. Valero, C. J. Cramer, D. G. Truhlar, J. Phys Chem. A 2009, 113, 5806-5812.

[49] P. E. Field, W. J. Green, J. Phys. Chem. 1971, 75, 821-825.

[50] W. J. Green, P. E. Field, J. Phys. Chem. 1980, 84, 3111-3114.

[51] D. Neiner, N. L. Okamoto, C. L. Condron, Q. M. Ramasse, P. Yu, N. D. Browning, S. M. Kauzlarich, J. Am. Chem. Soc. 2007, 129, 13857-13862.

Received: ((will be filled in by the editorial staff))

Revised: ((will be filled in by the editorial staff)) Published online: ((will be filled in by the editorial staff)) 
Semiconducting Clathrates Meet Gas Hydrates:

$\mathrm{Xe}_{24}\left[\mathrm{Sn}_{136}\right]$

Antti J. Karttunen and Thomas F. Fässler

Graphics and text for the Table of contents

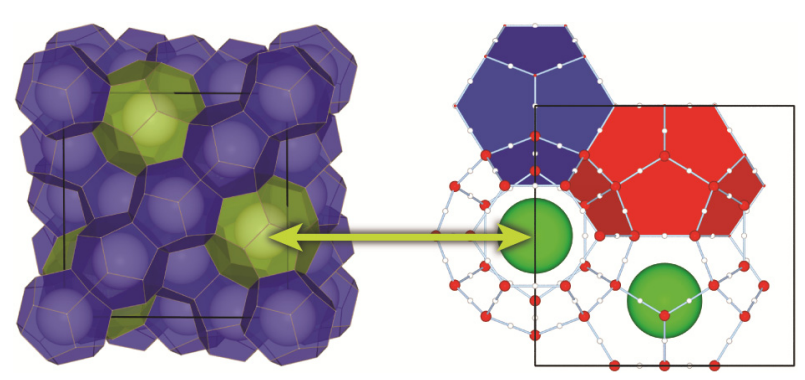

Combining the structural features of gas hydrates and semiconducting group 14 clathrates reveals a new class of porous semiconductor materials such as $\mathrm{Xe}_{24}\left[\mathrm{Sn}_{136}\right]$ 